\title{
The Quotient-Difference Algorithm and the Padé Table: An Alternative Form and a General Continued Fraction
}

\author{
By J. H. McCabe
}

\begin{abstract}
The quotient-difference algorithm is applied to a given power series in a modified way, and various continued fractions provided by the algorithm are described in terms of their relationships with the Pade table for the power series. In particular a general continued fraction whose convergents form any chosen combination of horizontal or vertical connected sequences of Padé approximants is introduced.
\end{abstract}

1. Introduction. In [4] Gragg gives a substantial review of some of the properties of the Padé table for a given power series $\sum_{k=0}^{\infty} c_{k} x^{k}$ and the relations between Padé approximants and other areas of numerical analysis. The earliest and, as Gragg suggests, perhaps the most significant of these related areas is the theory of continued fractions. In particular it is the so-called corresponding fractions for the power series that then provide the link between the Padé table and the quotient-difference algorithm of Rutishauser. One of the many applications of the quotientdifference algorithm is to obtain the coefficients of the corresponding fractions from those of the power series, and the convergents of these continued fractions form staircases in the Pade table for the series.

In this paper the quotient-difference algorithm is taken in a form that is slightly different from the usual and is then used to provide, very simply, the coefficients of six types of continued fractions whose convergents form ordered sequences of Padé approximants, including those mentioned above. It is then shown that the table of coefficients generated by the revised quotient-difference algorithm also provide the partial numerators and denominators of a 'general' continued fraction whose convergents are a sequence of Padé approximants that is made up of any chosen horizontal or vertical connected subsequences. This freedom in choosing the path in the Padé table means that some of the existing algorithms based on particular paths can be regarded as special cases of the revised quotient-difference algorithm. Two such instances are described.

The revised quotient-difference algorithm used in this paper is essentially the algorithm that has already been used to construct the continued fractions whose convergents form the so called two-point Pade table for two given power series, the only difference being the necessity for an additional rule for defining certain coefficients in the absence of a second series. However, by looking at the case where

Received February 24, 1982.

1980 Mathematics Subject Classification. Primary 10A30, 10F20, 30A22, 41A20.

Key words and phrases. Continued fractions, Padé approximants. 
some coefficients of a second series are available we see in the final section that the two point Padé table for two series is, in a sense, a limiting case of the usual Padé table for the sum of the two series.

2. The $q-d$ Algorithm and the Padé Table. The Padé table [see Figure 1] of the formal power series

$$
f(x)=\sum_{k=0}^{\infty} c_{k} x^{k}, \quad c_{0} \neq 0,
$$

is an infinite two-dimensional array of irreducible rational functions

$$
P_{n, m}(x)=\frac{\alpha_{0}+\alpha_{1} x+\cdots+\alpha_{m} x^{m}}{1+\beta_{1} x+\cdots+\beta_{n} x^{n}}, \quad m, n \geqslant 0,
$$

in each of which the coefficients are such that the expansion of $P_{n, m}(x)$ in powers of $x$ agrees with $f(x)$ as far as possible. The power series, and its associated Padé table, are said to be normal if, for each $m$ and $n$,

$$
P_{n, m}(x)=\sum_{k=0}^{m+n} c_{k} x^{k}+\text { higher order terms. }
$$

In this case every element of the table exists and differs from any other, and both $\alpha_{m}$ and $\beta_{n}$ are nonzero. A sufficient condition for the series to be normal is that the determinant

$$
\left[\begin{array}{lccl}
c_{r-s+1} & c_{r-s+2} & \cdots & c_{r} \\
c_{r-s+2} & c_{r-s+3} & \cdots & c_{r+1} \\
c_{r} & \cdots & & c_{r+s-1}
\end{array}\right]
$$

is nonzero for all $r$ and $s$, with $c_{k}=0$ if $k<0$. We will assume that the series (1) is normal.

$$
\begin{array}{ccccccc}
P_{0,0} & P_{1,0} & P_{2,0} & P_{3,0} & \ldots & P_{n, 0} & \ldots \\
P_{0,1} & P_{1,1} & P_{2,1} & P_{3,1} & \ldots & P_{n, 1} & \ldots \\
P_{0,2} & P_{1,2} & P_{2,2} & P_{3,2} & \ldots & P_{n, 2} & \ldots \\
\vdots & \vdots & \vdots & & & & \\
P_{0, m} & P_{1, m} & P_{2, m} & P_{3, m} & \ldots & P_{n, m} & \ldots
\end{array}
$$

Figure 1. The Padé table

The relationships between the Padé table for the series (1) and various continued fractions that correspond to the series are well known. There are many algorithms for constructing continued fractions whose convergents form ordered sequences in a normal Padé table. Perhaps the most fundamental of these is the quotient-difference algorithm of Rutishauser.

The coefficients of the series are used to provide the starting values

$$
q_{1}^{j}=c_{j+1} / c_{j}, \quad j \geqslant 0,
$$

for the recurrence relations

$$
\left.\begin{array}{l}
e_{i}^{j}=e_{i-1}^{j+1}+q_{i}^{j+1}-q_{i}^{j} \\
q_{i+1}^{j}=q_{i}^{j+1} * e_{i}^{j+1} \div e_{i}^{j}
\end{array}\right\}, \quad \begin{aligned}
& j=0,1,2, \ldots, \\
& i=1,2,3, \ldots,
\end{aligned}
$$


where $e_{0}^{j}=0$ for all values of $j$. These rhombus rules allow the calculation of those elements of the $q-d$ array on and below the diagonal with zero superfix, see Figure 2.

$\begin{array}{llllll}q_{1}^{0} & & q_{2}^{-1} & & q_{3}^{-2} & \cdots \\ & e_{1}^{0} & & e_{2}^{-1} & & \\ q_{1}^{1} & & q_{2}^{0} & & q_{3}^{-1} & \ldots \\ & e_{1}^{1} & & e_{2}^{0} & & \\ q_{1}^{2} & & q_{2}^{1} & & q_{3}^{0} & \ldots \\ & e_{1}^{2} & & e_{2}^{1} & & \\ q_{1}^{3} & & q_{2}^{2} & & q_{3}^{1} & \ldots \\ & e_{1}^{3} & & e_{2}^{2} & \cdot & \\ q_{1}^{4} & & q_{2}^{3} & \cdot & \cdot & \\ & e_{1}^{4} & \cdot & \cdot & \cdot & \\ q_{1}^{5} & \cdot & \cdot & . & & \\ \cdot & \cdot & \cdot & & & \\ \cdot & \cdot & & & & \\ . & & & & & \end{array}$

FIGURE 2. The q-d array

The staircase sequence of Padé approximants $P_{0, k}, P_{1, k}, P_{1, k+1}, P_{2, k+1}, P_{2, k+2}, \ldots$, are the successive convergents of the continued fraction

$$
c_{0}+c_{1} x+\cdots+\frac{c_{k} x^{k}}{1}-\frac{q_{1}^{k} x}{1}-\frac{e_{1}^{k} x}{1}-\frac{q_{2}^{k} x}{1}-\frac{e_{2}^{k} x}{1}-\frac{q_{3}^{k} x}{1}-\cdots
$$

for $k=0,1,2,3, \cdots$. The convergents of all these fractions thus provide those elements of the Padé table on and below the first upper para-diagonal. The remaining elements are the convergents of the continued fractions, of the form (5), that correspond to the reciprocal series of (1), i.e. series $\sum_{k=0}^{\infty} \check{c}_{k} x^{k}$, where

$$
\left(\sum_{k=0}^{\infty} c_{k} x^{k}\right)\left(\sum_{j=0}^{\infty} \check{c}_{j} x^{j}\right)=1 .
$$

The coefficients of these continued fractions, $\check{q}_{i}^{j}$ and $\check{e}_{i}^{j}$, can be obtained by first extending the $q$ - $d$ array for (1) to negative values of $j$ by using (4) and then

$$
\begin{aligned}
& q_{1}^{0}=-\check{q}_{1}^{0}, \\
& \check{q}_{i}^{j}=e_{i+j-1}^{1-j}, \quad \check{e}_{i}^{j}=q_{i+j}^{1-j},
\end{aligned}
$$

where the indices can take all values for which at least one of the elements involved is defined. These relations are given by Henrici [5]. Thus the $q-d$ algorithm (4) can provide continued fractions which in turn yield all the elements of the Pade table.

3. An Alternative Form of the $q$ - $d$ algorithm. Set $d_{1}^{j}=-c_{j} / c_{j-1}$ for $j=1,2, \ldots$, and use these ratios as starting values for the rhombus rules

$$
\left.\begin{array}{l}
n_{i+1}^{j}=n_{i}^{j+1}+d_{i}^{j+1}-d_{i}^{j} \\
d_{i}^{j+1}=n_{i}^{j+1} * d_{i-1}^{j} \div n_{i}^{j}
\end{array}\right\}, \quad j=1,2, \ldots,
$$


for $i=1,2,3, \ldots$, with $n_{1}^{j}=0$ for all $j$ and

$$
d_{i}^{1}=-n_{i}^{1},
$$

for $i=2,3,4, \ldots$. The elements generated form the $n$ - $d$ array shown in Figure 3.

$$
\begin{array}{llllll}
d_{1}^{1} & n_{2}^{1} & d_{2}^{1} & n_{3}^{1} & d_{3}^{1} & \ldots \\
d_{1}^{2} & n_{2}^{2} & d_{2}^{2} & n_{3}^{2} & d_{3}^{2} & \ldots \\
d_{1}^{3} & n_{2}^{3} & d_{2}^{3} & n_{3}^{3} & d_{3}^{3} & \ldots
\end{array}
$$

FIGURE 3. The $n$-d array

The convergents of the continued fraction

$$
c_{0}+c_{1} x+\cdots+\frac{c_{k} x^{k}}{1+d_{1}^{k} x}+\frac{n_{2}^{k} x}{1+d_{2}^{k} x}+\frac{n_{3}^{k} x}{1+d_{3}^{k} x}+\cdots,
$$

$k=1,2,3, \ldots$, are the Padé approximants $P_{1, k-1}, P_{2, k-1}, P_{3, k-1}, P_{4, k-1}, \ldots$, that is those on the $(k-1)$ th row of the Padé table. Continued fractions of the form (9) are called $M$ fractions for the series (1).

The above properties, with the exception of (8), follow from [8], where the even extensions of (9) are considered. The continued fraction (9) is the even part of

$$
c_{0}+c_{1} x+\cdots+\frac{c_{k} x^{k}}{1}+\frac{m_{1}^{k} x}{1}+\frac{l_{2}^{k}}{1}+\frac{m_{2}^{k} x}{1}+\frac{l_{3}^{k}}{1}+\frac{m_{3}^{k} x}{1}+\cdots,
$$

whose coefficients can be obtained by the further set of rhombus rules,

$$
\begin{aligned}
m_{i}^{j} * l_{i}^{j-1} & =l_{i}^{j} * m_{i-1}^{j-1}, \\
\left(1+l_{i}^{j}\right) * m_{i-1}^{j+1} & =\left(1+l_{i-1}^{j+1}\right) * m_{i-1}^{j}
\end{aligned}
$$

for $i, j=1,2, \ldots$, with $l_{1}^{j}=0, m_{1}^{j}=-c_{j} / c_{j-1}, j=1,2, \ldots$, and

$$
m_{i}^{1}=\frac{l_{i}^{1} * m_{i-1}^{1}}{1+l_{i-1}^{1}}, \quad i=2,3, \ldots
$$

See McCabe [8] for details. The continued fractions (10) are, in [8], called Perron fractions for the series (1), and the convergents of (10) are the sequence $P_{0, k}, P_{1, k-1}$, $P_{1, k}, P_{2, k-1}, P_{2, k}, P_{3, k-1}, P_{3, k}, \ldots$ in the Padé table.

The coefficients of the corresponding fractions (5), (9) and (10) can of course be expressed in terms of each other. Specifically

$$
\begin{aligned}
& n_{i}^{j}=-e_{i-1}^{j+1-i}, \\
& d_{i}^{j}=e_{i-1}^{j+1-i}-q_{i}^{j-i}
\end{aligned}
$$

for $i, j=1,2,3, \ldots$, and

$$
\begin{aligned}
l_{i}^{j} & =\frac{-n_{i}^{j}}{\left(n_{i}^{j}+d_{i-1}^{j}\right)}, \\
m_{i}^{j} & =\frac{d_{i}^{j} d_{i-1}^{j}}{\left(n_{i}^{j}+d_{i-1}^{j}\right)}
\end{aligned}
$$

for $i, j=1,2,3, \ldots$. 
Substituting for $l_{i}^{1}, m_{i-1}^{1}$ and $l_{i-1}^{1}$ from (14) in (12) yields

$$
m_{i}^{1}=\frac{-n_{i}^{1} d_{i-1}^{1}}{n_{i}^{1}+d_{i-1}^{1}}
$$

and equating this to (14b) with $j=1$ gives the result (8). The alternate version of the $q-d$ algorithm, as given by Eqs. (7), was first obtained in a study of two-point Padé approximations by McCabe [7]. The rhombus rules (11) were also given by Bussonnais [2].

The relations (13) are such that many of the characteristics of the quotient difference algorithm are retained by the alternate form. In particular the convergence of the $n$ columns of the $n$ - $d$ table to zero and of the $d$ columns to the inverse poles of $f(x)$, multiplied by -1 , will result if the columns of the $q-d$ array converge to zero and to the inverse poles of $f(x)$, respectively.

4. Two Further Continued Fractions. The convergents of the three forms of continued fraction encountered so far form, respectively, staircase sequences, row sequences and 'sawtooth' sequences in the Padé table, the latter being of the form

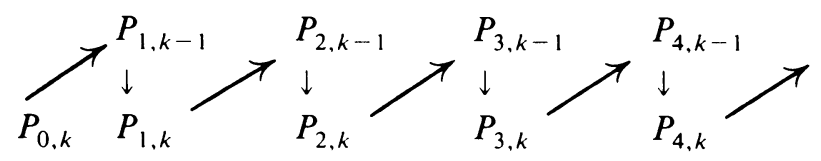

for some $k$.

Two additional continued fractions whose convergents form ordered sequences in the Padé table can be obtained by a simple re-ordering of the convergents of the Perron fraction (10). We have the following general result.

Let the convergents of the continued fraction

$$
\frac{a_{1}}{1}+\frac{a_{2}}{1}+\frac{a_{3}}{1}+\frac{a_{4}}{1}+\frac{a_{5}}{1}+\cdots
$$

be $A_{1} / B_{1}, A_{2} / B_{2}, A_{3} / B_{3}, \ldots$, and let the continued fraction whose convergents are those of $(15)$ with the $(4 k-1)$ th and $(4 k-2)$ th convergents interchanged for all $k \geqslant 1$, that is the sequence

$$
\frac{A_{1}}{B_{1}}, \frac{A_{3}}{B_{3}}, \frac{A_{2}}{B_{2}}, \frac{A_{4}}{B_{4}}, \frac{A_{5}}{B_{5}}, \frac{A_{7}}{B_{7}}, \frac{A_{6}}{B_{6}}, \frac{A_{8}}{B_{8}}, \ldots,
$$

be

$$
\frac{a_{1}^{1}}{b_{1}^{1}}+\frac{a_{2}^{1}}{b_{2}^{1}}+\frac{a_{3}^{1}}{b_{3}^{1}}+\frac{a_{4}^{1}}{b_{4}^{1}}+\cdots
$$

Then $a_{1}^{1}=a_{1}, b_{1}^{1}=1$ and

$$
\begin{array}{lll}
a_{4 k-3}^{1}=-a_{4 k-3} a_{4 k-4}, & b_{4 k-3}^{1}=1+a_{4 k-3}, & k>1, \\
a_{4 k-2}^{1}=a_{4 k-2}, & b_{4 k-2}^{1}=1+a_{4 k-1}, & k \geqslant 1, \\
a_{4 k-1}^{1}=-a_{4 k-1}, & b_{4 k-1}^{1}=1, & k \geqslant 1, \\
a_{4 k}^{1}=1, & b_{4 k}^{1}=a_{4 k}, & k \geqslant 1 .
\end{array}
$$


The left-hand relations imply that

$$
a_{4 k}^{1} \cdot a_{4 k-1}^{1} \cdot a_{4 k-2}^{1} \cdot a_{4 k-3}^{1}=a_{4 k-1} a_{4 k-2} a_{4 k-3} a_{4 k-4}
$$

for $k=2,3, \ldots$, while $a_{4}^{1} a_{3}^{1} a_{2}^{1} a_{1}^{1}=-a_{3} a_{2} a_{1}$. These relations are easily proved by induction based on the standard continued fraction results

$$
\frac{A_{k+1}^{1}}{B_{k+1}^{1}}-\frac{A_{k}^{1}}{B_{k}^{1}}=(-)^{k} \frac{a_{1}^{1} a_{2}^{1} a_{3}^{1} \cdots a_{k+1}^{1}}{B_{k}^{1} B_{k+1}^{1}}
$$

and

$$
\frac{A_{k+1}^{1}}{B_{k+1}^{1}}-\frac{A_{k-1}^{1}}{B_{k-1}^{1}}=(-)^{k+1} \frac{a_{1}^{1} a_{2}^{1} \cdots a_{k}^{1} b_{k+1}^{1}}{B_{k-1}^{1} B_{k+1}^{1}} .
$$

If we now apply this change of sequence to the convergents of the Perron fraction (10), we obtain the sequence of Padé approximants $P_{0, k}, P_{1, k}, P_{1, k-1}, P_{2, k-1}, P_{2, k}$, $P_{3, k}, P_{3, k-1}, P_{4, k-1}, \ldots$ The continued fraction whose convergents form this sequence is

$$
\begin{array}{r}
c_{0}+c_{1} x+\cdots+\frac{c_{k} x^{k}}{1}+\frac{m_{1}^{k} x}{1+l_{2}^{k}}-\frac{l_{2}^{k}}{1}+\frac{1}{m_{2}^{k} x}-\frac{l_{3}^{k} m_{2}^{k} x}{1+l_{3}^{k}}+\frac{m_{3}^{k} x}{1+l_{4}^{k}}-\frac{l_{4}^{k}}{1}+\frac{1}{m_{4}^{k} x} \\
\cdots-\frac{l_{2 j-1}^{k} m_{2 j-2}^{k} x}{1+l_{2 j-1}^{k}}+\frac{m_{2 j-1}^{k} x}{1+l_{2 j}^{k}}-\frac{l_{2 j}^{k}}{1}+\frac{1}{m_{2 j}^{k} x}-\cdots .
\end{array}
$$

By an equivalence transformation we can write this as

$$
c_{0}+c_{1} x+\cdots+\frac{c_{k} x^{k}}{1}+\frac{s_{1}^{k} x}{1}+\frac{t_{2}^{k}}{1}+\frac{s_{2}^{k}}{x}+\frac{t_{3}^{k} x}{1}+\frac{s_{3}^{k} x}{1}+\frac{t_{4}^{k}}{1}+\cdots
$$

where, provided that $l_{1}^{k}=0$,

$$
\begin{aligned}
t_{j}^{k} & =-l_{j}^{k} /\left(1+l_{j}^{k}\right), \\
s_{j}^{k} & =m_{j}^{k} /\left(1+l_{j}^{k}\right)\left(1+l_{j+1}^{k}\right), \quad j \text { odd } \\
& =1 / m_{j}^{k}, \quad j \text { even. }
\end{aligned}
$$

In terms of the coefficients generated by the alternative $q-d$ algorithm these simplify to

$$
\begin{aligned}
t_{j}^{k} & =n_{j}^{k} / d_{j-1}^{k}, \\
s_{j}^{k} & =n_{j+1}^{k}+d_{j}^{k}, \quad j \text { odd }, \\
& =\frac{n_{j}^{k}+d_{j-1}^{k}}{d_{j}^{k} d_{j-1}^{k}}, \quad j \text { even } .
\end{aligned}
$$

The convergents of (16) form a 'battlement' sequence in the Padé table, that is of the form

$$
\begin{aligned}
& P_{1, k-1} \rightarrow P_{2, k-1} \quad P_{3, k-1} \rightarrow P_{4, k-1} \\
& P_{0, k} \rightarrow \begin{array}{llllll}
\uparrow & P_{1, k} & P_{2, k} & \rightarrow & P_{3 k} & P_{4 k}
\end{array} \rightarrow
\end{aligned}
$$


The even part (or contraction) of the Perron fraction (10) is the $M$ fraction (9). Let us call this the first even part of the Perron fraction, in order to define the second even part as the continued fraction whose convergents are every second pair of those of (10), beginning with the third and fourth convergents. That is the convergents of the second even part are the 3rd, 4th, 7th, 8th, 11 th, 12 th, .. convergents of the Perron fraction. This fraction is of the form

$$
c_{0}+\cdots+\frac{c_{k} x^{k}}{1+g_{1}^{k} x}+\frac{h_{2}^{k} x^{2}}{1+g_{2}^{k} x}+\frac{h_{3}^{k}}{1+g_{3}^{k} x}+\frac{h_{4}^{k} x^{2}}{1+g_{4}^{k} x}+\cdots,
$$

and its convergents are the Padé approximants $P_{1, k}, P_{2, k-1}, P_{3, k}, P_{4, k-1}, \ldots$, and they form a second sawtooth sequence whose pattern is

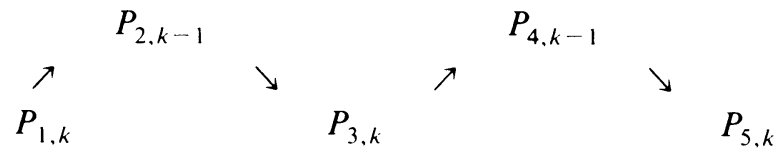

It is easily seen that the second even part of the Perron fraction (10) is the first even part of the continued fraction (16), and that the first even part of (16) is the second even part of the $M$ fraction (10). The standard relations between the ceofficients of a continued fraction and those of its first even part yield the coefficients of (17) as

$$
\begin{aligned}
h_{j}^{k} & =-t_{j}^{k} s_{j-1}^{k} / s_{j}^{k}=-n_{j}^{k} d_{j}^{k}, \quad j \text { even, } \\
& =-t_{j}^{k} /\left(1+t_{j}^{k}\right)=-n_{j}^{k} /\left(d_{j-1}^{k}+n_{j}^{k}\right), \quad j \text { odd }, \\
g_{j}^{k} & =\left(1-t_{j}^{k}\right) / s_{j}^{k}=d_{j}^{k}, \quad j \text { even, } \\
& =s_{j}^{k} /\left(1+t_{j}^{k}\right)=\left(n_{j+1}^{k}+d_{j}^{k}\right) d_{j-1}^{k} /\left(n_{j}^{k}+d_{j-1}^{k}\right) \\
& =d_{j}^{k+1}, \quad j \text { odd. }
\end{aligned}
$$

The continued fraction (17) has recently been shown to be applicable to the solution of the strong Hamburger moment problem, Sri Ranga [10].

5. A General Continued Fraction. The continued fraction (5), namely

$$
c_{0}+c_{1} x+\cdots+\frac{c_{k} x^{k}}{1}-\frac{q_{1}^{k} x}{1}-\frac{e_{1}^{k} x}{1}-\frac{q_{2}^{k} x}{1}-\frac{e_{2}^{k} x}{1}-\cdots
$$

or, equivalently,

$$
\begin{aligned}
c_{0}+c_{1} x+\cdots+ & \frac{c_{k} x^{k}}{1}+\frac{\left(n_{1}^{k+1}+d_{1}^{k+1}\right) x}{1}+\frac{n_{2}^{k+1} x}{1} \\
+ & \frac{\left(n_{2}^{k+2}+d_{2}^{k+2}\right) x}{1}+\frac{n_{3}^{k+2} x}{1}+\cdots,
\end{aligned}
$$

has convergents which form a staircase sequence in the Padé table, going from adjacent rows to adjacent columns, alternately. The table of coefficients provided by the rhombus rules (7) also provide the coefficients of the continued fraction whose convergents form any chosen path that is made up of horizontal and vertical steps, of any length, in the Padé table, including the special cases of row sequences entirely, or column sequences entirely. 
Suppose that, after a series of horizontal and/or vertical moves through the Pade table, we have arrived at the approximant $P_{i, j}$. We could have arrived there from either $P_{i-1, j}$ or $P_{i, j-1}$, and, whichever is the case, we can proceed to the approximant $P_{i+1, j}$ or the approximant $P_{i, j+1}$. Thus there are four possibilities for the set of three successive convergents centered on $P_{i, j}$, and in each case the three approximants are connected by the numerator and denominator of the next partial quotient in our continued fraction. The four possibilities, and the corresponding relation are

Case 1.

$$
\begin{gathered}
P_{i-1, j} \rightarrow P_{i, j} \rightarrow P_{i+1, j} \\
P_{i+1, j}(x)=\left(1+d_{i+1}^{j+1} x\right) P_{i, j}(x)+n_{i+1}^{j+1} x P_{i-1, j}(x)
\end{gathered}
$$

Case 2.

$$
\begin{array}{ccc}
P_{i-1, j} & \rightarrow & P_{i j} \\
& \downarrow \\
& P_{i, j+1} \\
P_{i, j+1}(x)=P_{i, j}(x)+n_{i+1}^{j+1} x P_{i-1, j}(x) .
\end{array}
$$

Case 3.

$$
\begin{gathered}
P_{i, j-1} \\
\downarrow \\
P_{i, j} \rightarrow P_{i+1, j} \\
P_{i+1, j}(x)=P_{i, j}(x)+\left(n_{i+1}^{j+1}+d_{i+1}^{j+1}\right) x P_{i, j-1}(x) .
\end{gathered}
$$

Case 4.

$$
\begin{gathered}
P_{i, j-1} \\
\downarrow \\
P_{i, j} \\
\downarrow \\
P_{i, j+1} \\
P_{i, j+1}(x)=\left(1-d_{i+1}^{j+1} x\right) P_{i, j}(x)+\left(n_{i+1}^{j+1}+d_{i+1}^{j+1}\right) x P_{i, j-1}(x), \quad j>0, \\
=\left(1-d_{i+1}^{j+1} x\right) P_{i, j}(x)-d_{1}^{1} d_{2}^{1} \cdots d_{i+1}^{1} x^{i+1}, \quad j=0 .
\end{gathered}
$$

In each case the numerators and denominators of the Padé approximants obey the relation separately. Any sequence of applications of the above steps will yield a continued fraction whose partial quotients are of the form $N x /(1+D x)$, where $N$ is always nonzero but $D$ can be zero. Some particular sequences are

(a) Repreated application of Case 1 with $j$ constant and $i$ taking the values $0,1,2, \ldots$ This yields the $M$ fraction (9) with convergents forming the $(j+1)$ th row of the Padé table $\left[P_{-1, j}\right.$ is taken as $1 / 0$ and $\left.n_{1}^{j+1}=c_{j+1} x^{j}\right]$.

(b) Application of Cases 3 and 2 alternately yields the continued fraction (18). [ $P_{i,-1}$ is taken as $0 / 1$ when necessary.]

(c) Repeated application of Case 4 with $i$ constant and $j=0$ initially yields a continued fraction whose convergents form the $(i+1)$ th column of the Pade table. 
For $i=0$ the resulting continued fraction is

$$
\begin{aligned}
\frac{1}{1}+\frac{d_{1}^{1} x}{1-d_{1}^{1} x}+\frac{d_{1}^{2} x}{1-d_{1}^{2} x} & +\frac{d_{1}^{3} x}{1-d_{1}^{3} x}+\cdots \\
= & \frac{1}{1}-\frac{c_{1} x / c_{0}}{1+c_{1} x / c_{0}}-\frac{c_{2} x / c_{1}}{1+c_{2} x / c_{1}}-\frac{c_{3} x / c_{2}}{1+c_{3} x / c_{2}}-\cdots,
\end{aligned}
$$

which is the familiar EQUIVALENT fraction for the given series (1).

The proof of Case 1 follows from [8] while Cases 2 and 3 are simple consequences of a result in [7]. Case 4 then follows as a consequence of the other three cases, as is now shown.

For simplicity we denote the approximant $P_{i, j}$ by $C$ and the other four approximants by $N, S, E$ and $W$, indicating their position relative to $C$ in a north, south, east and west sense. The first three relations are, figuratively,

$$
\begin{aligned}
& E=(1+d) C+n W, \\
& S=C+n W, \\
& E=C+(n+d) N,
\end{aligned}
$$

where $n$ and $d$ represent $n_{i+1}^{j+1} x$ and $d_{i+1}^{j+1} x$, respectively, and of course the relations hold for the numerators and denominators separately.

From (19) and (20) we see that

$$
S=E-d C
$$

and substituting for $E$ in (21) yields

$$
S=(1-d) C+(n+d) N
$$

as required.

We can also prove Wynn's identity,

$$
\frac{1}{N-C}+\frac{1}{S-C}=\frac{1}{E-C}+\frac{1}{W-C},
$$

but we have to consider both the numerators and denominators of the approximants at the same time, and so we write $E=E_{n} / E_{d}$ and a similar notation for the other approximants. For convenience we also write, for example, $[E, C]$ to mean $\left(E_{n} C_{d}-\right.$ $\left.E_{d} C_{n}\right)$ and so on.

Then, from (21)

$$
E_{n}-C_{n}=(n+d) N_{n} \text { and } E_{d}-C_{d}=(n+d) N_{d},
$$

and hence

$$
[E, C]=E_{n} C_{d}-E_{d} C_{n}=(n+d)\left(N_{n} C_{d}-N_{d} C_{n}\right)=(n+d)[N, C] .
$$

It thus follows that

$$
\frac{1}{E-C}=\frac{E_{d} C_{d}}{[E, C]}=\frac{E_{d} C_{d}}{(n+d)[N, C]}
$$

Similarly, from (20),

$$
\frac{1}{S-C}=\frac{S_{d} C_{d}}{n[W, C]} \text {. }
$$


The equations (19) and (21) combine to give

$$
W-C=\frac{n+d}{n}(N-C),
$$

and from this we obtain

$$
[W, C]=\frac{n+d}{n}[N, C]
$$

Thus

$$
\frac{1}{S-C}=\frac{S_{d} C_{d}}{(n+d)[N, C]},
$$

and

$$
\frac{1}{W-C}=\frac{n W_{d} C_{d}}{(n+d)[N, C]} .
$$

Finally,

$$
\frac{1}{N-C}=\frac{N_{d} C_{d}}{[N, C]},
$$

and so

$$
\begin{aligned}
& \frac{1}{E-C}+\frac{1}{W-C}-\frac{1}{S-C}-\frac{1}{N-C} \\
& =\frac{C_{d}}{(n+d)[N, C]} \cdot\left\{E_{d}+n W_{d}-S_{d}-(n+d) N_{d}\right\} .
\end{aligned}
$$

But (20) subtracted from (21) gives

$$
E_{d}-S_{d}=(n+d) N_{d}-n W_{d},
$$

and the proof is complete.

Baker's algorithms (see [1]) for computing the elements of the Padé table are

$$
P_{n, m}=P_{n-1, m+1}-\frac{a_{n-1, m+1} x P_{n-1, m}}{a_{n-1, m}}
$$

and

$$
P_{n, m-1}=\frac{a_{n-1, m} P_{n, m}-a_{n, m} P_{n-1, m}}{a_{n-1, m}-a_{n, m}}
$$

where $a_{i j}$ is the leading coefficient of the numerator of $P_{i, j}$ and, once again, the numerators and denominators of the Padé approximants obey the rules separately. From (22) we find

$$
E=S+d C,
$$

and identifying this with the first of Baker's algorithms, that is setting $C$ to be $P_{n-1, m}$, yields

$$
d_{n}^{m+1}=-\frac{a_{n-1, m+1}}{a_{n-1, m}} .
$$

Then, by equating (19) and (21), we obtain

$$
N=\frac{n}{n+d} C+\frac{d}{n+d} W,
$$


and identifying this with the second of Baker's algorithms, that is setting $C$ to be $P_{n, m}$, yields

$$
\frac{n_{n+1}^{m+1}}{n_{n+1}^{m+1}+d_{n+1}^{m+1}}=-\frac{a_{n, m}}{a_{n-1, m}-a_{n, m}}, \quad \frac{d_{n+1}^{m+1}}{n_{n+1}^{m+1}+d_{n+1}^{m+1}}=\frac{a_{n-1, m}}{a_{n-1, m}-a_{n, m}}
$$

or

$$
n_{n+1}^{m+1}=-\frac{a_{n, m} d_{n+1}^{m+1}}{a_{n-1, m}}=\frac{a_{n, m+1}}{a_{n-1, m}}
$$

since

$$
d_{n+1}^{m+1}=-a_{n, m+1} / a_{n, m} .
$$

A study of Gragg's algorithm, see [4], which computes the elements lying on an ascending staircase in the Pade table, shows that the continued fraction involved is that which results from using the relations

$$
E=S+d C
$$

and

$$
N=\frac{n}{n+d}(C+W)
$$

alternately.

The continued fraction (17) provides relations between approximants that occupy the relative positions $S W, C$ and $S E$ in the Pade table, while the even contraction of the continued fraction (18), namely an associated continued fraction of the form

$$
c_{0}+c_{1} x+\cdots+\frac{c_{k} x^{k}}{1+u_{1}^{k} x}+\frac{u_{1}^{k} x^{2}}{1+v_{2}^{k} x}+\frac{u_{3}^{k} x^{2}}{1+v_{3}^{k} x}+\cdots
$$

has convergents which form a diagonal or off-diagonal of the Padé table. It should be a simple process to allow our general continued fraction to travel in northeasterly or south-westerly directions in the Padé table.

6. Stability. It is well known that the quotient-difference algorithm can be unstable, the problems arising because of the formation of differences in the $q$-columns. Rutishauser [9] provides some examples demonstrating instability. Gargantini and Henrici [3] studied the stability problem when the algorithm is used to calculate the coefficients of continued fractions, and they tried several possible approaches to overcome instability, only one of which was successful to any extent.

It is inevitable that the stability problems will also arise when the above modified quotient-difference algorithm is used, the equations (13) linking the two algorithms indicate that this will be the case.

Recently however a method of performing the arithmetic of the quotient-difference algorithm without the tendency to amplify the errors has been given by Stokes [11]. The same approach can be used on the modified algorithm, and Stokes demonstrates his method by applying it to the calculation of continued fraction coefficients. In particular, he uses it to produce continued fractions approximating series expansions for a function about two points. From the remarks in the final section of this paper it will be seen that this is a particularly relevant application. 
7. Examples. As a first example, consider the series expansion

$$
\begin{aligned}
\sqrt{(1+2 x)}= & 1+x-\frac{x^{2}}{2}+\frac{x^{3}}{2}-\frac{5 x^{4}}{8} \\
& +\cdots+(-)^{k-1} \frac{1 \cdot 3 \cdot 5 \cdots(2 k-3) x^{k}}{k !}+\cdots .
\end{aligned}
$$

The initial part of the $n-d$ array is

\begin{tabular}{l|rrrrr}
$j$ & $d_{1}^{j}$ & $n_{2}^{j}$ & $d_{2}^{j}$ & $n_{3}^{j}$ & $d_{3}^{j}$ \\
\hline 1 & -1 & $3 / 2$ & $-3 / 2$ & $5 / 3$ & $-5 / 3$ \\
2 & $1 / 2$ & $1 / 2$ & $-1 / 3$ & $5 / 6$ & $-3 / 4$ \\
3 & 1 & $1 / 4$ & $1 / 4$ & $1 / 2$ & $-1 / 5$ \\
4 & $5 / 4$ & $3 / 20$ & $3 / 5$ & & \\
5 & $7 / 5$ & & & &
\end{tabular}

In this case the coefficients can be expressed explicitly by

$$
d_{i}^{j}=\frac{(2 j-2 i+1)(2 j-2 i-1)}{j(2 j-1)-(i-1)(2 i-1)}
$$

and

$$
n_{i}^{j}=\frac{(2 j-2 i+1)(2 j-2 i+3)(i-1)(2 i-1)}{\{j(2 j-1)-(i-2)(2 i-3)\}\{j(2 j-1)-(i-1)(2 i-1)\}} .
$$

It is easily verified that, when $j=1$,

$$
d_{i}^{1}=-n_{i}^{1}=\frac{1-2 i}{i} .
$$

An example of a family of power series, for which just over half of the elements of the $n$ - $d$ array can be expressed explicitly, is the hypergeometric series

$$
{ }_{2} F_{1}(1, \lambda ; 1+\omega ;-z)=\sum_{r=0}^{\infty} \frac{(\lambda)_{r}}{(1+\omega)_{r}}(-z)^{r},
$$

were $\omega$ is not a negative integer. The series is absolutely convergent if $|z|<1$, divergent if $|z|>1$, while if $|z|=1$ the series converges absolutely if $\omega>1$.

The elements on and below the staircase formed by coefficients $d_{k}^{k}, n_{k+1}^{k}$ for $k=1,2,3, \ldots$, are given by

$$
d_{i}^{j}=\frac{\lambda+j-1}{\omega+i+j-1}, \quad j \geqslant i, i=1,2,3, \ldots,
$$

and

$$
n_{i}^{j}=\frac{(i-1)(i-1+\omega-j)}{(\omega+i+j-2)(\omega+i+j-1)}, \quad j \geqslant i-1, i=2,3,4, \ldots .
$$

It does not appear possible to express the remaining coefficients in such a way except for particular values of $\lambda$ and $\omega$.

As a final example, consider the function

$$
F(x, p)=e^{-x^{p}} \int_{0}^{x} e^{t^{p}} d t, \quad x \geqslant 0, p>0 .
$$


This is a generalization of Dawson's integral, obtained when $p=2$, which occurs in various branches of physics. Setting $u=x^{p}$ and $\alpha=1 / p$ allows the expansion of $F(x, p)$ as the convergent series

$$
F(x, p)=x \sum_{k=0}^{\infty} \frac{(-u)^{k}}{(1+\alpha)_{k}} .
$$

The starting values for the $n-d$ array are thus $d_{1}^{j}=1 /(j+\alpha)$. For $p=2$ the initial part of the $n-d$ array is

\begin{tabular}{l|llllr}
$j$ & $d_{1}^{j}$ & $n_{2}^{j}$ & $d_{2}^{j}$ & $n_{3}^{j}$ & \multicolumn{1}{c}{$d_{3}^{j}$} \\
\hline 1 & $2 / 3$ & $-4 / 15$ & $4 / 15$ & $-2 / 21$ & $2 / 21$ \\
2 & $2 / 5$ & $-4 / 35$ & $2 / 7$ & $-8 / 63$ & $16 / 45$ \\
3 & $2 / 7$ & $-4 / 63$ & $2 / 9$ & $-8 / 99$ & $2 / 11$ \\
4 & $2 / 9$ & $-4 / 99$ & $2 / 11$ & & \\
5 & $2 / 11$ & & & &
\end{tabular}

One of the continued fractions that is obtainable for Dawson's integral is thus

$$
F(x, 2)=\frac{x}{1}+\frac{2 x^{2} / 3}{1}-\frac{4 x^{2} / 15}{1}+\frac{6 x^{2} / 35}{1}-\cdots,
$$

whose convergents form the main staircase of the Padé table of the series expansion.

The function $F(x, p)$ also has the divergent asymptotic expansion at the point at infinity

$$
F(x, p) \sim \alpha x \sum_{r=0}^{\infty}(1-\alpha)_{r} / u^{r+1}, \quad u=x^{p} \rightarrow \infty .
$$

The alternative form of the quotient-difference algorithm can be used to generate the coefficients of continued fractions which correspond to both series expansions simultaneously, and whose convergents are the so-called two-point Padé approximants. These rational functions are discussed briefly in the next section. An example of such a continued fraction expansion is

$$
F(x, p)=\frac{\alpha x}{\alpha+x}-\frac{u}{\alpha+1-u}-\frac{2 u}{\alpha+2+u}-\frac{3 u}{\alpha+3+u}-\cdots
$$

Details of the properties of this expansion when $p=2$ are given in [7].

8. The Two-Point Padé Table. The alternative form of the quotient-difference algorithm (7) first arose out of a study of the continued fractions associated with the two-point Padé table, the points being the origin and the point at $\infty$. See [7] for details. Briefly, suppose, in addition to the series (1), we also have the series

$$
\sum_{k=1}^{\infty} \frac{b_{k}}{x^{k}}, \quad b_{1} \neq 0
$$

in which for some $N \geqslant 1, b_{k}=0$ for all $k>N$. Set $d_{1}^{0}=c_{0} / b_{1}$ and $d_{1}^{-j}=-b_{j} / b_{j+1}$ for $j=1,2, \ldots, N-1$, and then use the rhombus rules (7), with $d_{i}^{1-N}=-n_{i}^{1-N}$, $i=2,3, \ldots$, to generate the coefficients $n_{i}^{j}$ and $d_{i}^{j}, i=2,3,4, \ldots, j=1-N$, 
$2-N, \ldots, 0,1,2, \ldots$ The $r$ th convergent of the continued fraction

$$
\begin{aligned}
c_{0}+c_{1} x+\cdots \frac{c_{k} x^{k}}{1+d_{1}^{k} x} & +\frac{n_{2}^{k} x}{1+d_{2}^{k} x}+\frac{n_{3}^{k} x}{1+d_{3}^{k} x} \\
+ & \frac{n_{4}^{k} x}{1+d_{4}^{k} x}+\cdots, \quad k \geqslant 0,
\end{aligned}
$$

is a rational function which agrees with $(r+k)$ terms of $(1)$ and $(r-k)$ terms of (30) when expanded accordingly. It is easily established that, for $k>0$ and $r \leqslant k$, the $r$ th convergent of (31) is the Pade approximant $P_{r \cdot k-1}$ for the series (1). In the special case $k=0$, the $r$ th convergent of (31) is a ratio of polynomials of degrees $(r-1)$ and $r$, respectively; it agrees with $r$ terms of each of the series (1) and (30) when expanded accordingly. Such continued fractions have been used by Thron, Jones and Waadeland [6] to solve the strong Stieltjes moment problem.

For $0<k<N$ the $r$ th convergent of the continued fraction

$$
\frac{b_{1}}{x}+\frac{b_{2}}{x^{2}}+\cdots-\frac{b_{k} / x^{k}}{1+d_{1}^{-k} x}+\frac{n_{2}^{-k} x}{1+d_{2}^{-k} x}+\frac{n_{3}^{-k} x}{1+d_{3}^{-k} x}+\cdots
$$

agrees with $(r+k)$ terms of (30) and $(r-k)$ terms of (1) when expanded accordingly.

Finally, it is easily seen that the table of coefficients

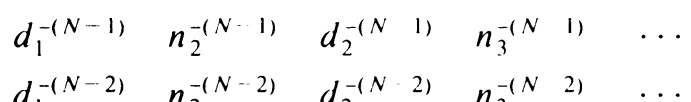

$$
\begin{aligned}
& \left.d_{1}^{-(N-2)} \quad n_{2}^{-(N-2)} \quad d_{2}^{-(N-2)} \quad n_{3}^{-(N} 2\right) \quad \cdots \\
& \begin{array}{lllll}
d_{1}^{0} & n_{2}^{0} & d_{2}^{0} & n_{3}^{0} & \ldots \\
d_{1}^{1} & n_{2}^{1} & d_{2}^{1} & n_{3}^{1} & \ldots
\end{array}
\end{aligned}
$$

in which $d_{i}^{-(N-1)}=-n_{i}^{-(N-1)}, i=2,3, \ldots$, has the same relation to the Padé table for the series

$$
\frac{1}{b_{N}}\left\{b_{N}+b_{N-1} x+\cdots+b_{1} x^{N-1}-\sum_{j=0}^{\infty} c_{j} x^{j+N}\right\}
$$

as the table in Figure 3 has to the Pade table for the series $\sum_{j=0}^{\infty} c_{j} x^{j}$. That is, for $N$ finite, the two-point Pade table for the two series is the Padé table for the sum of the two series.

When $N$ is not finite the rhombus rules (7) are used to provide the coefficients of (31) and (32) for all $k \geqslant 0$. The convergents of these continued fractions are the elements of the doubly infinite two-point Padé table for the series. All the previous continued fraction expansions relate to the two-point Padé table and have the described paths through it. The correspondence properties of these expansions with the two series follow from the structure of the two-point Pade table, described in [7]. 
Acknowledgement. The author is grateful to the referee for his helpful comments, resulting in improvements to the initial draft of this paper.

Department of Applied Mathematics

University of St. Andrews

St. Andrews, Fife, Scotland KY16 9SS

1. G. A. Baker, JR., "The Padé approximant method and some related generalizations," The Padé Approximant in Theoretical Physics (G. A. Baker, Jr. and J. L. Gammel, eds.), Academic Press, New York, 1970. pp. 1-39.

2. D. Bussonnais, Tous les Algorithms de Calcal par Recurrence des Approximations de Padé d'une Serie. Construction des Fractions Continues Correspondantes, Conference on Padé Approximation, Lille, 1978 .

3. I. Gargantini \& P. Henrici, "A continued fraction algorithm for the computation of higher transcendental functions in the complex plane," Math. Comp., v. 21, 1967, pp. 18-29.

4. W. B. Gragg, "The Pade table and its relation to certain algorithms of numerical analysis," SIAM Rev., v. 14, 1972, pp. 1-62.

5. P. Henrici, "Quotient-difference algorithms," Mathematical Methods for Digital Computers, Vol. 11 (A. Ralston and M. S. Wilf, eds.), Wiley, New York, 1967, pp. 35-62.

6. W. B. Jones, W. A. Thron \& H. Waadeland, “A strong Stieltjes moment problem," Trans. Amer. Math. Soc., v. 261, 1980, pp. 503-528.

7. J. H. MCCABE, "A formal extension of the Padé table to include two-point Pade quotients," J. Inst. Math. Appl., v. 15, 1975, pp. 363-372.

8. J. H. MCCABE, "Perron fractions: an algorithm for computing the Pade table," J. Comp. Appl. Math., v. 7. 1981, pp. 271-275.

9. H. Rutishauser, "Der quotienten-differenze algorithmus," Mittlg. Inst. fur Angew. Math., ETH, nr 7. Birkhäuser-Verlag. Berlin, 1956.

10. A. SRI Ranga, The Strong Hamburger Moment Problem, Internal Report, University of St. Andrews, 1982.

11. A. N. Stokes, “A stable quotient difference algorithm," Math. Comp., v. 34, 1980, pp. 515-519. 